\begin{tabular}{|c|c|c|c|c|c|}
\hline MUNIBE Antropologia-Arkeologia & $n^{\circ} 71$ & $59-71$ & DONOSTIA & 2020 & ISSN 1132-2217 • eISSN 2172-4555 \\
\hline
\end{tabular}

\title{
Dix mille ans de peuplement humain sur le versant nord des Pyrénées (21000-11000 cal BP) entre unification et régionalisation culturelles
}

\author{
Ten thousand years of human settlement in the northern \\ slope of the Pyrenees (21000-11000 cal BP) \\ between cultural unification and regionalisation
}

MOTS-CLÉS: Paléolithique récent et final, Pyrénées, Magdalénien, Azilien, Laborien, équipements lithiques, industrie osseuse, littoral, peuplement. GAKO-HITZAK: Paleolitiko berria eta amaierakoa, Pirinioak, Madeleine aldia, Azil aldia, laboriarra, ekipamendu litikoak, hezur-industria, kostaldea, populazioa. KEY WORDS: Recent and Final Palaeolithic, Pyrenees, Magdalenian, Azilian, Laborian, Lithic Tool-kit, Osseous Industry, Coastline, Settlement.

\section{Mathieu LANGLAIS ${ }^{(1,3)}$, Célia FAT CHEUNG(2), Véronique LAROULANDIE(1) Alexandre LEFEBVRE(2), Benjamin MARQUEBIELLE ${ }^{(2)}$, Jean-Marc PÉTILLON ${ }^{(2)}$}

\section{RÉSUMÉ}

Les dix derniers millénaires d'occupation humaine paléolithique ont laissé d'abondants témoignages le long du versant nord-pyrénéen. Si les fluctuations climatiques et leurs conséquences environnementales ont pu conditionner l'exploitation de certains territoires en altitude, le piémont semble en revanche avoir constitué un vaste espace favorable à l'implantation humaine entre des reliefs et vallées progressivement libérées des glaces, l'ouverture de la plaine aquitaine, les littoraux atlantiques et méditerranéens. À partir de travaux récemment aboutis ou en cours, et d'ensembles de sites clés révisés, nous proposons une synthèse sur les principales évolutions des équipements lithiques et osseux et sur des comportements originaux (économie littorale, consommation des petits gibiers) entre 21000 et 11000 cal BP. Les différents segments chronoculturels sont représentés de façon très inégale. Au-delà de ce constat, nous évoquerons les raisons possibles de cette situation contrastée (conservation des sites, état de la recherche ou réalité de peuplement). II s'agira finalement de mieux comprendre ses variations internes et ses interactions avec le Bassin aquitain et le nord de la Péninsule ibérique mais également avec les rivages atlantiques et méditerranéens.

\section{LABURPENA}

Paleolitikoko azken hamar milurtekoetako giza okupazioek lekukotasun ugari utzi dute Pirinioen ipar-isurialdean barna. Klima-gorabeherek eta haiek ingurumenean izaniko ondorioek baliteke baldintzatu izana altueran dauden zenbait lurralderen ustiapena; aldiz, mendi-oinetako lautadak espazio zabal egokiak izan dira gizakiak egonkortzeko, hala nola izotzetatik apurka-apurka gabetutako ibarren eta erliebeen artean, Akitania aldeko lautada zabaletan, bai eta Atlantikoko zein Mediterraneoko kostaldeetan ere. Orain dela gutxi egindako edo oraindik abian diren lanetatik eta berrikusitako hainbat gako gunetatik abiaturik, ekipamendu litikoek eta hezur-lanabesek izaniko eboluzio nagusiaren laburpen bat proposatzen dugu, baita jokabide originalei buruzkoa ere (ibai-ekonomia, ehiztari txikien kontsumoa), 21.000 eta 11.000 oraina baino lehen. Segmentu kronokultural batzuk eta besteak oso era ezberdinean irudikatzen dira. Ondorio horretatik harago, egoera kontrastatu honen arrazoi posibleak aipatuko ditugu (kokalekuen kontserbazioa, ikerketaren egoera edo populazioaren errealitatea). Azken buruan, asmoa izango da hobeto ulertzea haien aldaerak, eta ikustea zer harreman izan duten Akitaniako arroarekin eta Iberiar penintsulako iparraldearekin, baita Atlantikoko eta Mediterraneoko itsasertzekin ere.

\section{ABSTRACT}

From the start of the Magdalenian to the end of the Laborian, ten millennia of human occupation have provided abundant evidence along the north Pyrenean slope. Climatic fluctuations (from GS-2b to GS-1), glacial history and their environmental consequences may have influenced the exploitation of certain altitude territories. In contrast, the foothills seem to have constituted a favourable area between reliefs and valleys gradually freed from ice, the opening Aquitaine plain, the Atlantic and Mediterranean coasts. Based on recently completed or ongoing work, and revised key sites or sets of sites, we propose here a synthesis to review the main changes in lithic and osseous equipment and original behaviours (coastal economy, use of small game) between 21000 and 11000 cal BP. However, the different moments of this evolution are represented in a very uneven way. Beyond that, we will discuss the possible reasons of the statement (conservation of the sites, state of research or settlement data). Among the privileged sectors, the Arudy basin is the scene of recent field operations and revaluations of collections. Other sites, such as Belvis, Gouërris, Troubat or Rhodes II, have benefited from PhD research (lithic and bone industries, archaeofauna, chronology, etc.). Data on the early

\footnotetext{
(1) Laboratoire PACEA UMR 5199, CNRS, univ. Bordeaux. Bâtiment B2, Allée Geoffroy Saint Hilaire CS 5002333615 Pessac cedex. mathieu.langlais@u-bordeaux.fr, veronique.laroulandie@u-bordeaux.fr

(2) TRACES UMR 5608, CNRS, univ. Toulouse-Jean-Jaurès. Maison de la Recherche 5, allées Antonio Machado 31058 Toulouse cedex 9 jean-marc.petillon@univ-tlse2.fr, fatcheung.celia@gmail.com, alexandrelefebvre24@gmail.com, benjamin.marquebielle@yahoo.fr (3) SERP, universitat de Barcelona. Carrer de Montalegre, 6 Barcelona 08001, Espagne.
} 
Magdalenian period remain limited. With the exception of certain sites assigned to this period from dates C14 onwards, only the altitude site of Montlleó makes it possible to testify to an increase in altitude as early as post-Late Glacial Maximum period. The Early Middle Magdalenian, mainly documented by ancient excavations, which are difficult to re-evaluate from an archaeostratigraphic point of view, remains poorly understood in the Pyrenees. Nevertheless the presence of certain objects establish links with Aquitaine and the Cantabrian Sea. The Recent Middle and Upper Magdalenian are particularly well documented periods in the Pyrenees. These occupations illustrate, in particular, a development of occupations in the context of often decorated cave bottoms and the anthropization of valleys freed from ice with hunting camp to ibex. The Azilian region has significant regional economic variations with a decline of craftsmanship in the bone industry and a technical simplification of lithic production. The Laborian is documented by the revaluation of old series provides evidence of Aquitaine region influences but in stratigraphically complicated contexts. Beyond the question of the unity of the Pyrenean chain between Magdalenian and Laborien, it will finally be a question of better understanding its interactions with the Aquitaine basin and the north of the Iberian Peninsula but also to the Atlantic and Mediterranean coastlines.

\section{INTRODUCTION}

Le versant nord de l'isthme pyrénéen a joué un rôle structurant dans le peuplement et la mobilité des groupes nomades de la fin du Paléolithique entre 21000 et 11000 cal BP (e.g. Lacombe, 1998; Dachary, 2002; Pétillon, 2006; Langlais, 2007; Barbaza, 2011; Fat Cheung, 2015). Frontière biologique relative pour certaines espèces, comme le renne (voir synthèse dans Lefebvre, 2016), le massif pyrénéen a contraint le territoire des chasseurs-collecteurs mais n'a pour autant jamais été imperméable aux flux d'objets, d'idées et de groupes humains durant cette période (e.g. Utrilla et Mazo, 1996; Cazals et al. dir., 2007; Corchón et al., 2009; Langlais, 2010; Sauvet et al., 2014; Pétillon et al. 2015; Sánchez de la Torre, 2015; Langlais et al. 2016, Pétillon et al. 2017; Mangado dir., 2018; Lefebvre et al., sous presse). Rappelons que nous avons d'ailleurs récemment interrogé la notion de «frontière préhistoriographique» à partir de l'exemple du Magdalénien moyen récent pyrénéen et des divergences terminologiques de part et d'autre de la frontière politico-linguistique actuelle (Langlais et Pétillon, 2019).

Au regard des modèles chronoculturels récemment proposés dans le nord du Bassin aquitain ou dans les Cantabres (Pétillon et al. ed., 2016; Straus et Langlais dir., 2020), quels sont les scénarios d'évolution des communautés humaines du Paléolithique récent et final sur le versant nord des Pyrénées? Considérant le nord de l'isthme pyrénéen compris entre l'océan Atlantique et la mer Méditerranée, et non strictement réduit au massif, notre objectif est ici de dresser un bilan sur le peuplement humain et sur les trajectoires culturelles régionales afin de s'interroger sur les discontinuités géographiques, chronologiques et techniques de ce vaste domaine.

D'un point de vue très général, entre 21000 et 11000 cal BP, la synchronie des changements environnementaux et culturels demeure délicate à appréhender du fait de la carence d'archives locales précises (Jalut et al., 1988 et 1992), tant environnementales (quelques enregistrements non connectés) qu'archéologiques (problème des fouilles anciennes et/ou partielles). Cette période, longue de dix millénaires, est marquée par des changements climatiques majeurs: la fin du Dernier Maximum glaciaire ou DMG (seconde partie du GS-2.1b de la chronologie glaciaire, voir Rasmussen et al. 2014), l'événement d'Heinrich-1 (cf. GS-2.1a), le réchauffement du Gl-1 et un retour du froid sec (GS-1) avant l'Holocène. Parmi les transformations liées à ces changements climatiques, l'ouverture d'espaces nouvellement accessibles dans le massif pyrénéen, où les glaciers de vallée se retirent progressivement dès la fin du DMG (Calvet et al., 2011; Delmas, 2015), a pu jouer un rôle essentiel dans le peuplement pyrénéen à cette période.

L'étude intégrée de plus d'une centaine de séries lithiques et osseuses ainsi qu'un cadre radiométrique renouvelé (dates 14C sur objets ciblés) construit à partir de plusieurs projets collectifs (p.e.: projet ANR Magdatis, projet Région Aquitaine Magdaqui, PCR PAVO), permettent de proposer et de comparer les stratotypes culturels détaillés pour le nord du Bassin aquitain et le massif pyrénéen (Langlais et Pétillon, 2019; Langlais, 2020b: fig. 1). Il existe des chevauchements chronologiques en partie liés à la résolution du cadre radiométrique mais qui posent aussi la question de la variabilité régionale de ces structurations. En se focalisant sur le versant nord pyrénéen sensu lato (du pays basque au sud de la Montagne noire), il est remarquable de constater que certaines étapes du modèle aquitain manquent à l'appel ou sont sous-documentées (fig. 1). Nous ne reviendrons donc ici pas en détail sur les données archéologiques précises, renvoyant alors aux publications, pour nous focaliser sur les tendances évolutives dans le temps long.

\section{QUEL(S) SCÉNARIO(S) DE PEUPLEMENT DES PYRÉNÉES ENTRE 21000 ET 11000 CAL BP?}

\subsection{Un «Magdalénien inférieur» peu (ou mal) documenté}

Autour de 21000 cal BP, le technocomplexe à lamelles à dos dextre marginal, contemporain de la transition Badegoulien - Magdalénien (Ducasse et al., 2011; Ducasse et Langlais, 2019; Langlais, 2020a), est attesté dans la grotte des Scilles en Haute-Garonne, site fouillé anciennement et réévalué il y a une dizaine d'années (Pétillon et al., 2008; Langlais et al., 2010). Le cadre radiométrique de cette phase de transition est actuellement en cours de révision dans le projet DEX_TER (S. Ducasse et $\mathrm{M}$. Langlais coord.).

Entre 20500 et 19500 cal BP, le Magdalénien inférieur à microlamelles à dos est documenté dans seulement trois gisements aux marges des Pyrénées (fig. 2): 


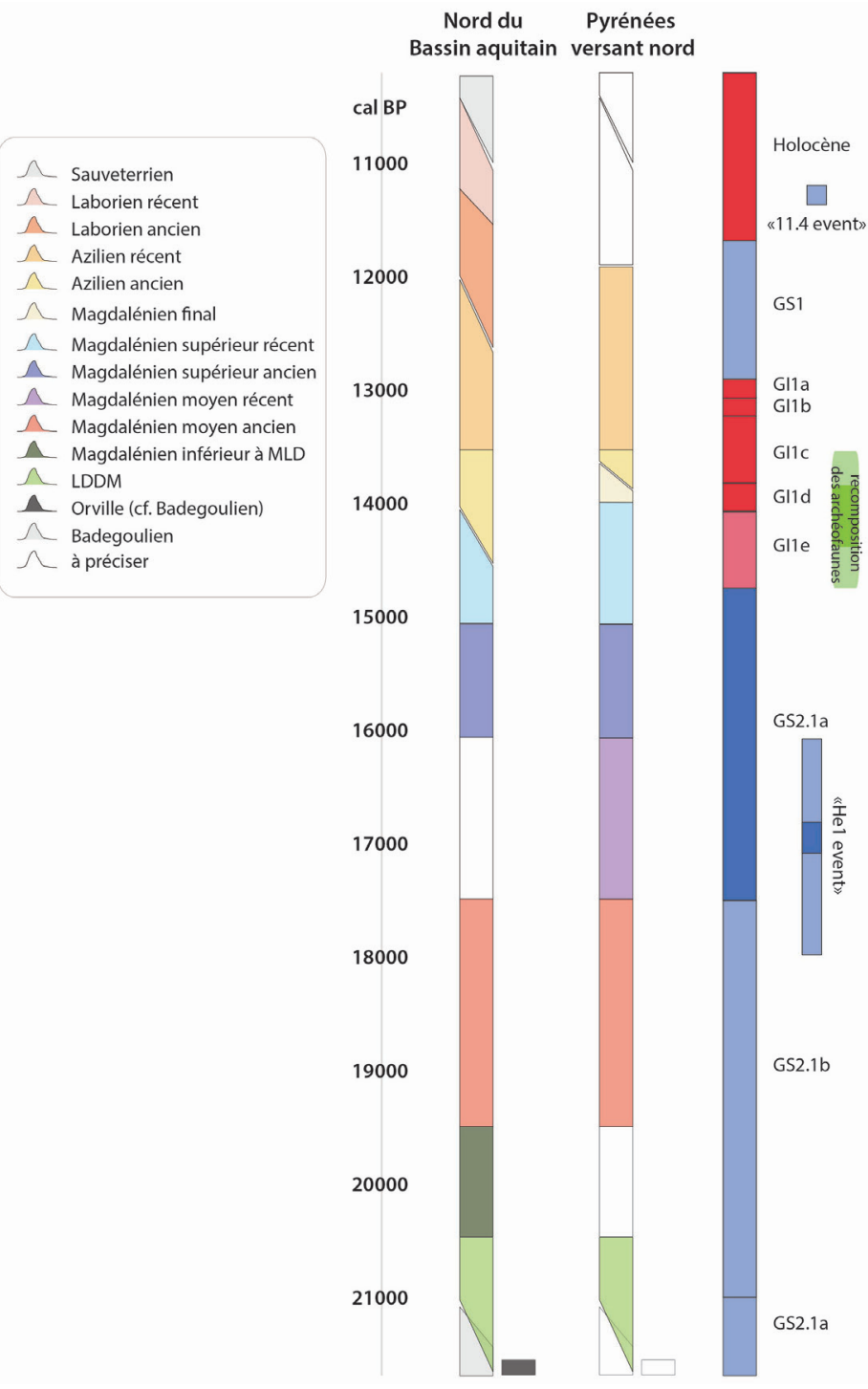

Fig.1. Comparaison des évolutions culturelles modélisées en stratotypes entre le nord du Bassin aquitain et le versant nord des Pyrénées (LDDM: lamelles à dos dextre marginal, MLD: microlamelles à dos; d'après Langlais 2020b; données climatiques d'après Rasmussen et al., 2014). / Comparison of cultural evolutions modeled in stratotypes between the northern part of the Aquitaine Basin and the northern slope of the Pyrenees (from Langlais, 2020b, climatic data from Rasmussen et al., 2014).
Montlleó, au sud du massif, dans la vallée de la Cerdagne, en Catalogne (Langlais, 2007, 2010; Mangado dir., 2018); et vers l'ouest, dans le Pays Basque: Erralla (Altuna et al., 1985; Cazals 2000) et Ekain (Altuna et Merino 1984). Dans ce dernier site, l'ensemble à microlamelles est en contexte de mélange stratigraphique (Cazals et Langlais 2005). L'attribution chronoculturelle du Rec del Penjat, dans les Pyrénées-Orientales, demeure délicate (Langlais, 2007). Des dates radiocarbone compatibles avec le Magdalénien inférieur à microlamelles à dos sont disponibles à Laa 2 c.5 dans les Pyrénées-Atlantiques (Pétillon et al., 2017) et à Lassac dans l'Aude (Pétillon et Ducasse, 2012). Toutefois, ces dates sont associées respectivement à des assemblages archéologiques peu diagnostiques ou non individualisés. Au regard de ce faible corpus et des biais de diagnose, il est difficile d'appréhender des variations internes au sein du versant nord des Pyrénées durant cette période.
Seul le site frontalier de plein air de Montlleó, au sud du versant, localisé à 1140 m d'altitude, en cours de fouilles depuis près de vingt ans sous la direction de membres du laboratoire SERP de l'université de Barcelone (X. Mangado, J.M. Fullola, O. Mercadal †, M. Sánchez de la Torre) apporte de nouvelles données. Il livre une stratigraphie originale où se distinguent deux ensembles (fig. 3). Au sommet, se trouve le Magdalénien inférieur à microlamelles à dos, daté autour de 20000 - 19000 cal BP. II repose sur un ensemble contenant quelques pointes à cran et des raclettes qui pourraient suggérer un mélange «solutréo-badegoulien» et, audelà, tisser des liens avec l'Espagne méditerranéenne (Mangado dir., 2018; Sánchez de la Torre et al., 2019).

\subsection{Un «Magdalénien moyen ancien» encore flou}

Le Magdalénien moyen ancien est reconnu sur un vaste espace européen depuis les Cantabres jusqu'en 


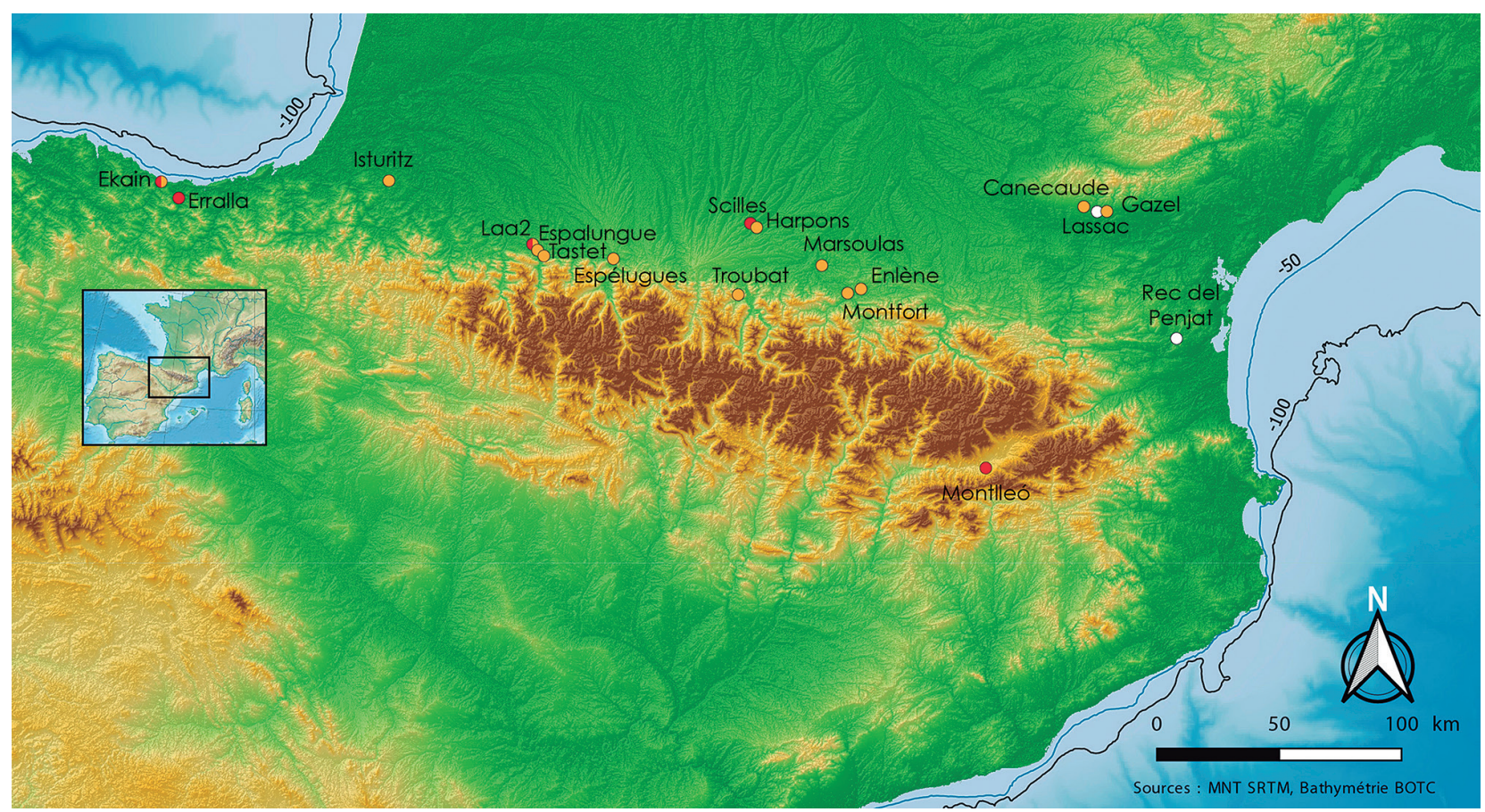

Fig.2. Localisation des sites du Magdalénien inférieur (ronds rouges: principaux sites; ronds blancs: attribution discutée) et du Magdalénien moyen ancien (ronds oranges), fond de carte F. Baleux, CAO ML. / Location of the main sites of Lower Magdalenian (red circles; white circles: discussed allocation) and Early Middle Magdalenian (orange circles), map F. Baleux, CAD ML.

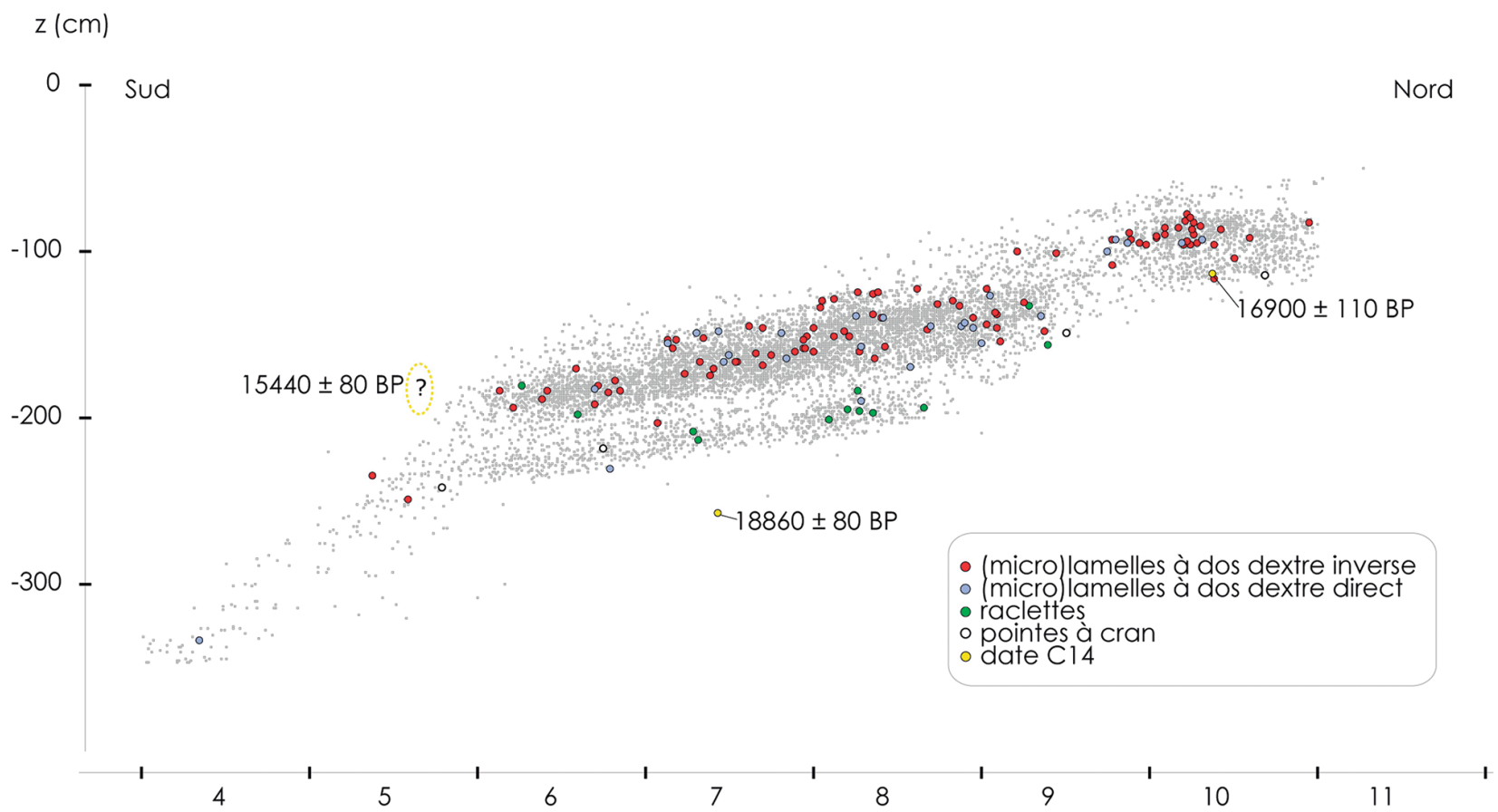

Fig.3. Projection stratigraphique sur un plan sagittal des silex coordonnés et des marqueurs typologiques et chronologiques du Secteur B de Montlleó (d'après Langlais, 2020b). / Sagittal stratigraphic scatterplot of coordinated flints and markers from Montlleó secteur B (from Langlais, 2020b).

Pologne (voir synthèse dans Sécher, 2017). Sur le versant nord des Pyrénées (fig. 2), sa diagnose repose essentiellement sur la présence de pointes en bois de cervidé à biseau simple de type «Lussac-Angles»
(Pétillon et Averbouh, 2013; Langlais et al., 2017), dont certaines sont datées directement comme à Isturitz (Pétillon, 2016a). D'autres sites dépourvus de cette armature osseuse livrent des dates radiocarbone 
contemporaines du Magdalénien moyen ancien. Cette situation indique une difficulté de diagnose du Magdalénien moyen ancien dans la région, notamment due à l'ancienneté des fouilles, mais soulève aussi la question de la variabilité du Magdalénien moyen ancien dans le domaine nord-pyrénéen (Langlais, 2010; Pétillon et al., 2017). Rappelons néanmoins que des liens matérialisés par certains objets particuliers sont tissés entre le Bassin aquitain, les Pyrénées et les Cantabres (Sécher, 2020). On peut notamment citer la grotte d'Enlène qui livre plusieurs marqueurs du Magdalénien moyen ancien (Bégouën et al. dir. 2019). Les travaux menés à la grotte Tastet, dans le bassin d'Arudy, dirigés par l'un d'entre nous (JMP), permettront prochainement de documenter un ensemble daté du Magdalénien moyen ancien à partir de fouilles récentes.

\subsection{Le Magdalénien moyen récent: densifica- tion du réseau nord-pyrénéen}

Le Magdalénien moyen récent représente un temps fort du Paléolithique récent nord-pyrénéen avec de nombreux sites révélés à partir de fouilles anciennes et modernes, voire en cours (fig. 4). À l'échelle de la moitié ouest de la France, la répartition des sites datés du Magdalénien moyen récent permet d'observer une discontinuité à l'échelle du peuplement magdalénien. En effet, autour de 17000 cal BP, les sites sont absents de la plaine nord-aquitaine jusque vers le nord du seuil du Poitou (Barshay-Szmidt et al., 2016). Cette lacune ne résultant pas de l'état de la recherche (éventuel biais de prospection, d'identification, etc.), il semble donc que ces zones aient été marginalisées à cette période. Il est alors possible que des populations

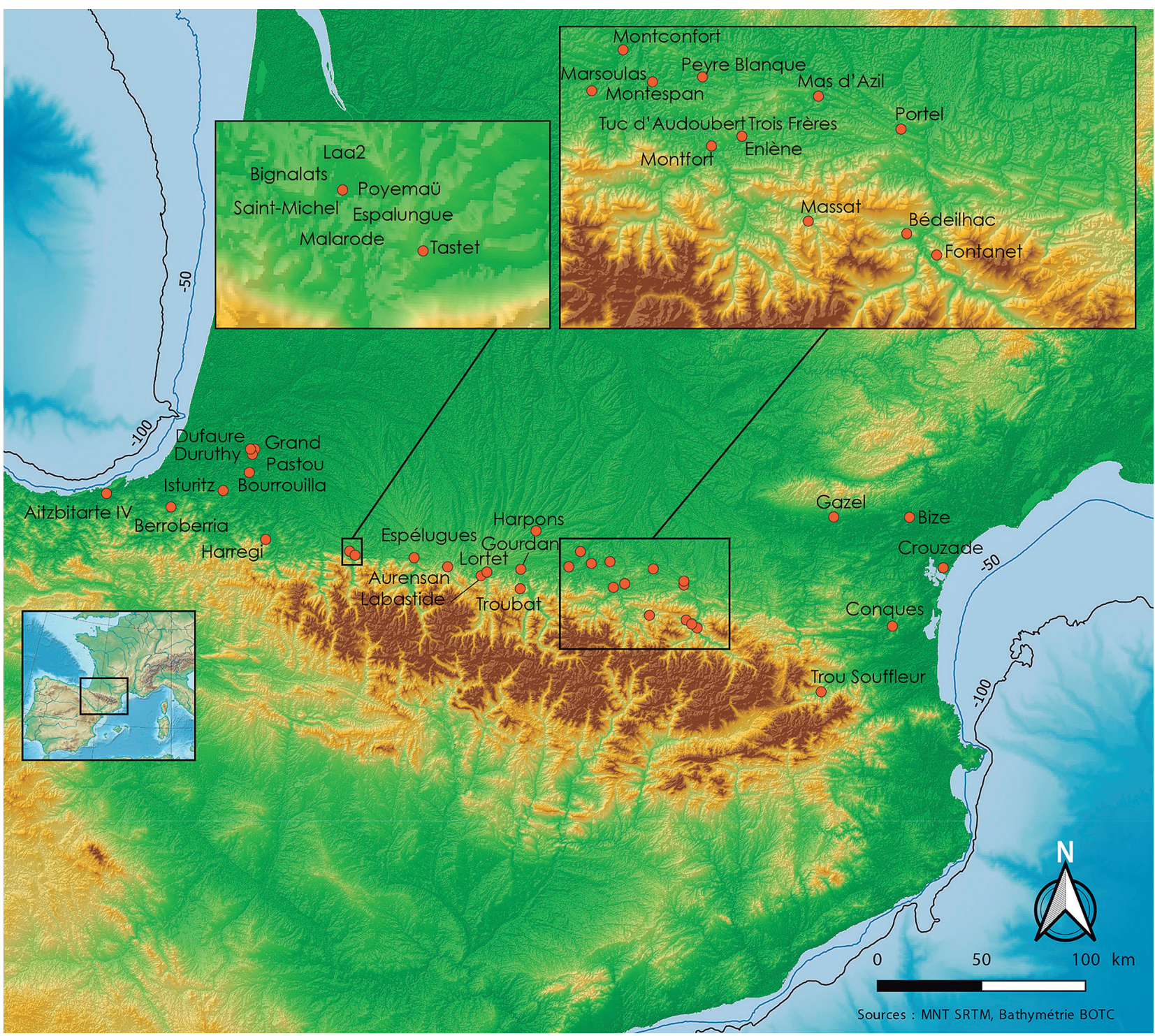

Fig.4. Localisation des principaux sites du Magdalénien moyen récent (fond de carte F. Baleux, CAO ML). / Location of the main sites of Recent Middle Magdalenian (map F. Baleux, CAD ML). 
se soient repliées dans des espaces aux topographies plus contrastées, le piémont pyrénéo-cantabrique constituant alors le cœur de l'aire culturelle du Magdalénien moyen récent. Le rôle des effets climatiques et environnementaux de l'évènement de Heinrich 1 dans cette situation reste à préciser (Pétillon et al., 2016; Laroulandie et al., 2017).

Parmi les comportements culturels originaux de cette période pour le versant nord-pyrénéen, citons la valorisation des grandes lames en silex. Elle se signale à la fois: 1) par la haute technicité des débitages normalisés et productifs et l'apprentissage des savoir-faire qu'elle sous-entend, 2) par la circulation des lames sur de grandes distances, leur utilisation comme support d'outil à longue durée de vie ou de nucléus à lamelles, mais aussi 3) par leur présence dans des dépôts en contexte de fond de grotte ornée (Langlais et al., 2016; Langlais, 2020b). Ce comportement singulier est particulièrement bien documenté dans les Hautes-Pyrénées et en Ariège, comme à Labastide, au Mas d'Azil et à Enlène (Simonnet, 1982), mais aussi dans les Pyrénées-Atlantiques, à Tastet (Langlais et Pétillon, 2019), voire à Saint-Michel d'Arudy pour des supports de nucléus à lamelles (Langlais obs. pers.). II s'agit de grandes lames en silex (15-25 cm de long), provenant en particulier du Campanien supérieur du Bergeracois, dont les sources sont localisées à environ 200-250km au nord. Dans l'industrie osseuse, à côté de l'apparition de toute une série de nouvelles parures (e.g. contours découpés de têtes animales bien documentés dans l'espace pyrénéo-cantabrique: voir Buisson et al., 1996; Bégouën et al. dir., 2009 et 2019), le Magdalénien moyen récent est un moment de fort développement des décors gravés et sculptés qui envahissent les équipements. Comme pour les grandes lames, la signification culturelle de ces objets socialement investis nous échappe.

\subsection{Une forte implantation au Magdalénien su- périeur}

À la différence de l'Aquitaine, où des séries livrent des morphotypes de pointes lithiques aisément différenciables et permettant de sérier le Magdalénien supérieur en différentes phases (Langlais, 2018), nous appréhendons le Magdalénien supérieur du versant nord des Pyrénées comme un seul ensemble, bien que certains morphotypes osseux permettent de nuancer cela (notamment les pointes à base fourchue, présentes à la charnière entre Magdalénien moyen récent et Magdalénien supérieur mais disparaissant ensuite: Pétillon, 2006). Au sein de toute la chaine pyrénéenne, comme pour le Magdalénien moyen récent, le Magdalénien supérieur sensu lato a été fortement recherché et fouillé depuis le début du XXe s., ce qui aboutit à une carte de sites très dense (fig. 5). Une certaine unité se distingue à travers la présence de petits triangles scalènes allongés dans plusieurs gisements comme
Belvis (900 m d'altitude) dans l'Aude (Sacchi, 1986), Troubat dans les Hautes-Pyrénées (Barbaza, 1996) et pour le sud du massif, dans les sites d'El Parco en Catalogne (Langlais, 2007) ou de Chaves en Aragon (Jimenez, thèse en cours). Les tableaux de chasse riches en bouquetins pourraient illustrer une exploitation accrue des contreforts montagneux. En revanche, les sites localisés dans la partie occidentale des Pyrénées (Dachary, 2002) - Duruthy, Dufaure ou Tizon sont clairement en connexion avec le Bassin aquitain comme l'indiquent certains objets lithiques particuliers commes des pointes à cran et burins de Lacan (Langlais, 2018).

Le Magdalénien supérieur du sud-ouest de la France en général, et du versant nord-pyrénéen en particulier, est également le théâtre d'une recomposition progressive des grandes faunes (Costamagno et al., 2016). Dans certains sites, le cerf est très bien représenté (Bourrouilla, Troubat; ibid.). Pourtant, le bois de renne est toujours le matériau privilégié pour l'industrie osseuse sur le versant nord du massif (Lefebvre, 2016). Cela implique donc des stratégies d'exploitation intégrant pleinement l'anticipation des besoins, comme à Troubat voire sur le versant sud en Catalogne à la Bora Gran d'En Carreras (fig. 6).

Parallèlement au nombre important de sites attribués au Magdalénien supérieur sur le versant nord des Pyrénées, la consommation de petits gibiers (poissons et oiseaux notamment) est bien documentée (Costamagno et Laroulandie, 2004). Concernant plus particulièrement les oiseaux, la partie occidentale livre plusieurs assemblages du Magdalénien supérieur contenant des restes de chouette harfang (Bubo scandiacus: voir synthèse dans Laroulandie, 2016). Cet espace correspond probablement à la limite méridionale de la niche écologique de ce rapace. Dans le Pays basque, le site de Santa Catalina livre également du harfang, cette fois-ci associé à de nombreux Anatinés et à plusieurs espèces d'oiseaux marins comme le grand pingouin (Pinguinus impennis) et des goélands (Larus hyperboreus / marinus et L. gr. Argentatus) (Laroulandie et al., 2016). Vers l'Est, le spectre avifaunique chassé est composé surtout de lagopèdes (Lagopus sp.) et de chocards (Pyrrhocorax graculus). Si, à Gazel (Aude), lagopèdes et chocards sont consommés dès le Magdalénien moyen récent, c'est au cours du Magdalénien supérieur que la chasse aux oiseaux semble se développer dans le versant nord des Pyrénées. L'acquisition de ce nouveau gibier procure divers produits: matière carnée, os longs, griffes, plumes. Outre les adaptations et les nouveaux savoir-faire qu'elle implique, elle s'accompagne sans doute d'un changement dans la manière dont les chasseurs ont pensé et appréhendé leur environnement et les ressources qui s'y trouvaient. Pour l'Azilien et le Laborien, les données sont rares mais de tels comportements ont été relevés notamment à Troubat et Gazel (voir synthèse dans Laroulandie, 2007). 

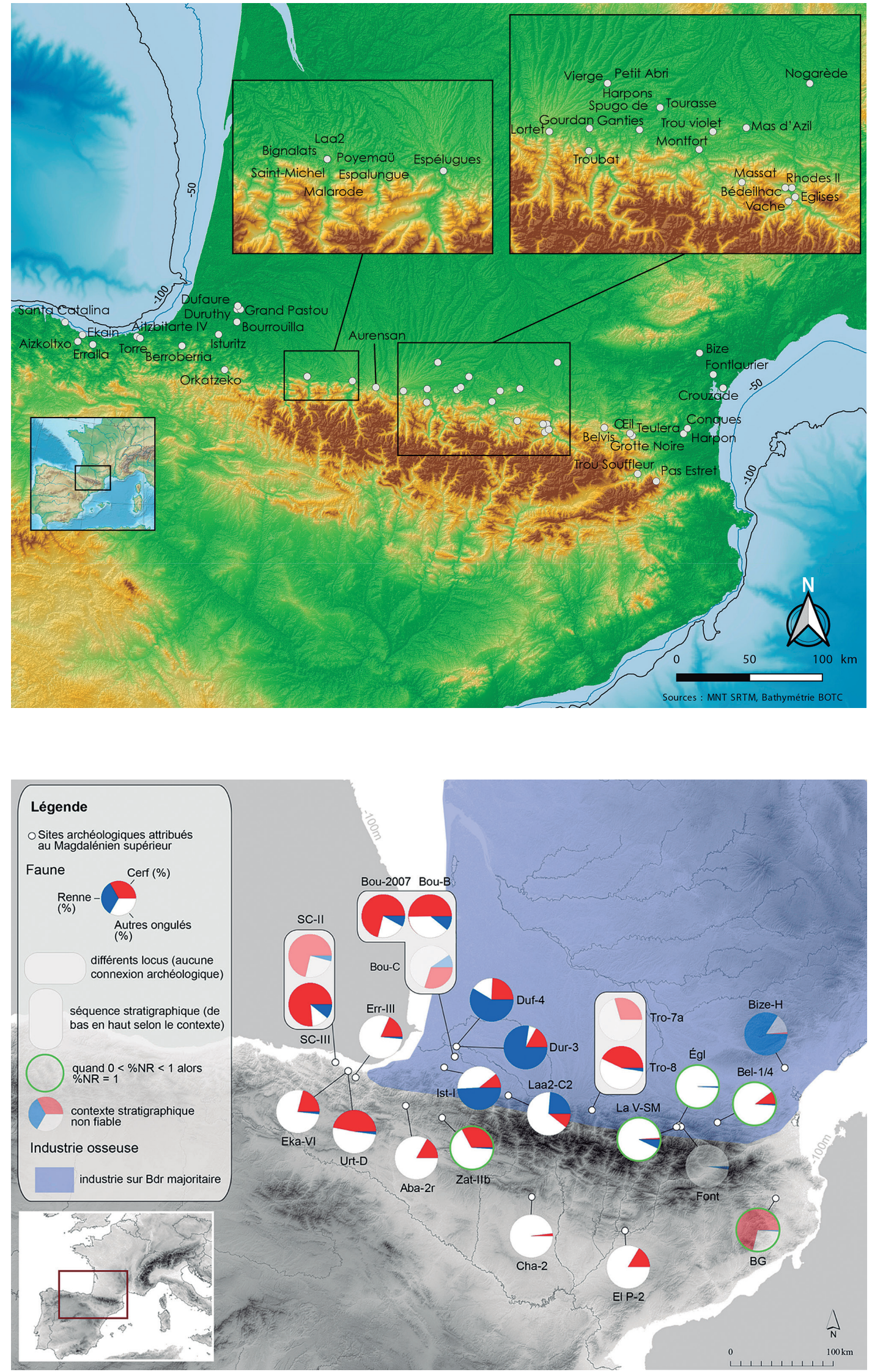

Fig.5. Localisation des principaux sites du Magdalénien supérieur, (fond de carte F. Baleux, CAO $\mathrm{ML})$. / Location of the main sites of Upper Magdalenian (map F. Baleux, CAD ML)
Fig.6. Distribution du cerf et du renne dans les spectres de cervidés chassés et dans l'industrie sur bois de cervidé de plusieurs sites du Magdalénien supérieur pyrénéen (Bou: Bourrouilla, SC Santa Catalina, Eka: Ekain, Urt: Urtiaga, Aba: Abauntz, Zat: Zatoya, Ist: Isturitz, Cha: Chaves, El P: Parco, BG: Bora Gran, Font: Fontanet, La V-SM: La Vache Salle Monique, Bel: Belvis, Égl: Les Églises, Tro: Troubat, Dur: Duruthy, Duf: Dufaure: CAO AL).I Distribution of deer and reindeer in hunted game spectrum and in antler industry from several sites in the Upper Magdalenian from Pyrenees (CAD AL). 


\subsection{L’Azilien: originalités nord-pyrénéennes}

Le peuplement azilien est bien documenté sur le versant nord du massif (fig. 7). Toutefois, par rapport au modèle aquitain (Langlais, 2020b) le processus de transformation - progressive ou rapide - des comportements culturels du Magdalénien vers une nouvelle identité azilienne apparait plus complexe sur le versant nord des Pyrénées (e.g. Barbaza et Lacombe, 2005; Martzluff, 2009; Barbaza, 2011; Fat Cheung, 2015). Dans la lignée des travaux de R. Simonnet (1967), la réévaluation de la séquence de Rhodes II permet de souligner un phasage interne de l'Azilien, dont la première phase présente néanmoins des différences avec ce qui caractérise I'Azilien ancien aquitain (Fat Cheung, 2015; Fat Cheung et Langlais, sous presse). En revanche, la séquence de Troubat pourrait témoigner d'une perduration tardive de comportements du Magdalénien alors que caractérise I'Azilien classique apparait plus tardif (Barbaza, 1996 et 2011). Parallèlement à la raréfaction du travail des matières osseuses, qui demeure essentiellement représenté par des poinçons en os et des têtes de harpon en bois de cerf (e.g. Marquebielle, 2018), et à un resserrement fonctionnel des outillages lithiques autour des pièces esquillées, grattoirs, couteaux et pointes à dos, nous pouvons rappeler le phénomène de galets gravés ou peints bien documenté dans l'Azilien classique pyrénéen (e.g. Couraud, 1985; Fat Cheung, 2015). Rappelons qu'en Andorre, à la Balma Margineda, un ensemble clairement azilien est daté autour de 13500 cal BP (Martzluff 2005).
Le processus d'azilianisation original du versant nord des Pyrénées (Fat Cheung, 2015 et 2020) diffère des modèles aquitain ou jurassien/alpin (Mevel, 2017; Tomasso et al., 2018; Naudinot et al., 2019). Cependant, il ne concerne qu'une partie de la chaîne montagneuse: en effet, à l'ouest et jusqu'en Pays Basque, une continuité est perceptible avec le modèle aquitain (séries à pointes à dos sur petites lames normalisées et outils laminaires à retouches écailleuses attribuées au Magdaleniense final: e.g. Armendariz, 1994; Barandiaran et Cava, 1994; Utrilla et Laborda, 2018; Soto et al., 2015). Dans la partie centrale des Pyrénées, bien que l'hypothèse d'une perduration tardive du Magdalénien soit fondée sur de rares sites (Troubat notamment) mais sans observation d'interstratifications avec l'Azilien, les prémices de l'Azilien ne concerneraient pas cette zone géographique (e.g. Barbaza, 1996; Fat Cheung, 2015).

Le second temps est représenté par un Azilien récent commun à l'ensemble du versant nord-pyrénéen, mais présentant néanmoins des variations techno-économiques (soin différentiel dans la production laminaire, exploitation des roches tenaces). Seule la séquence de Rhodes II pourrait nourrir I'hypothèse d'une variation chronologique interne à l'Azilien dans les Pyrénées centrales. Vers l'Est, les sites sont bien moins fréquents et une influence méditerranéenne est sensible avec ce qui est dénommé l'Epimagdaleniense en Catalogne notamment (Roman et al., 2016).

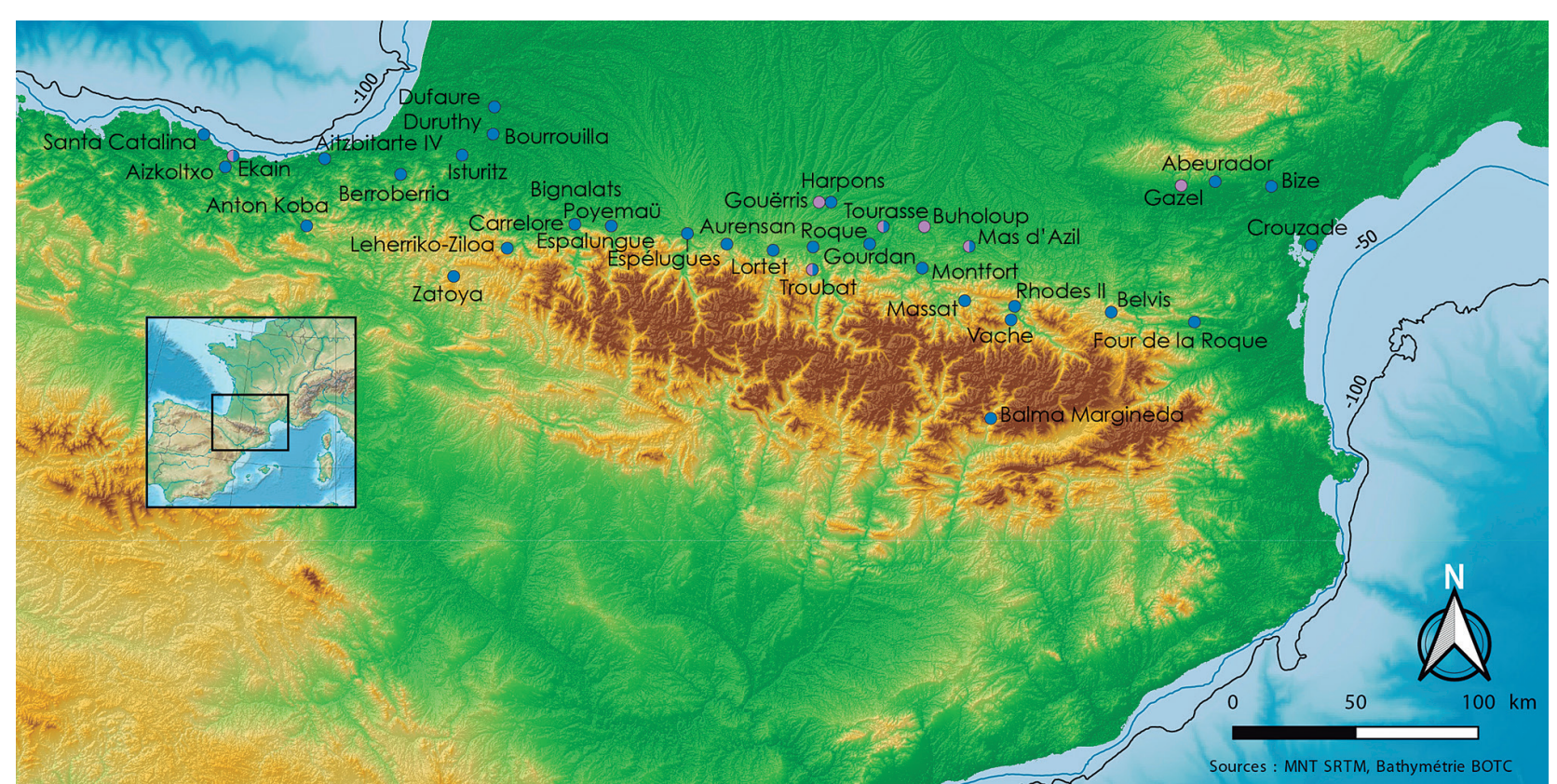

Fig.7. Localisation des principaux sites de l'Azilien (ronds bleus) et du Laborien (ronds mauves); fond de carte F. Baleux, CAO ML./ Location of the main sites of Azilian (blue circles) and Laborian (purple circles); map F. Baleux, CAD ML. 


\subsection{Du Laborien dans les Pyrénées!}

Dernier segment chronocuturel de la trajectoire proposée ici (Langlais, 2020b), le Laborien est longtemps demeuré fantomatique sur le versant nord des Pyrénées. À la lumière des travaux menés récemment en Aquitaine (Langlais et al., 2019), on peut penser qu'il s'agit ici aussi d'une sous-évaluation ou d'un hiatus documentaire. En effet, la révision de séries aziliennes (Fat Cheung, 2015) a souligné la présence d'éléments caractéristiques du Laborien mélangés au sein de plusieurs séquences aziliennes (fig. 7). Cette diagnose est fondée notamment sur la présence de pointes de Malaurie et d'un changement technique dans la composante laminaire, en rupture avec l'Azilien récent nord-pyrénéen. Le Laborien est ainsi documenté en Haute-Garonne à Gouërris c.B (Fat Cheung et Marquebielle, en prep.) et sur le site de Buholoup c. 7 et 8 (Briois et Vaquer, 2009). Les autres contextes sont moins bien connus car ces indices se trouvent dans des couches dont le contexte est perturbé, comme à Troubat couche 5 et quelques indices dans la couche 6 (Fat Cheung, 2015). La Tourasse et le Mas d'Azil livrent également des pointes de Malaurie typiques dans des contextes potentiels de mélange avec l'Azilien (ibid.). L'examen du matériel lithique des couches 5 et 6 de Gazel (Sacchi, 1986) a conduit à proposer une attribution au Laborien (Langlais, 2010). À l'autre extrémité de la chaîne, la couche 2 d'Ekain (Altuna et Merino, 1984) s'en rapproche également d'après la description des armatures (Fat Cheung, 2015).

\subsection{Bilan}

En définitive, l'espace nord pyrénéen mêle des originalités et des discontinuités qui peuvent être interprétées tantôt comme des hiatus documentaires, tantôt comme des trajectoires régionales particulières. Un «retour au local» s'avère incontournable afin de mieux apprécier la synchronicité des changements environnementaux et culturels et mesurer la plasticité des comportements humains dans ces environnements contrastés. À l'instar des prospections et fouilles réalisées par M. Conkey et S. Lacombe en Ariège, ou bien des opérations en cours au Mas d'Azil (M. Jarry, F. Bon, C. Pailler, L.-A. Lelouvier), à Sorde-l'Abbaye (J.-C. Merlet) et à Arancou (M. Dachary), nous pouvons souligner l'apport des recherches collectives menées dans le bassin d'Arudy (Pétillon et al., 2015 et 2017).

\section{DISCUSSIONS: LE VERSANT NORD-PYRÉNÉEN APRĖS LE DMG, UN MASSIF INTERCONNECTÉ}

Si les fluctuations climatiques (du GS-2.1b au GS1) et leurs conséquences environnementales ont pu conditionner l'exploitation de certains territoires en altitude, le piémont nord-pyrénéen semble en revanche avoir constitué après le DMG un écotone favorable aux chasseurs-collecteurs entre des reliefs et vallées progressivement libérés des glaces, l'ouverture de la plaine aquitaine et les littoraux atlantique et méditerranéen.

\subsection{L'isthme nord-pyrénéen: un trait d'union entre deux littoraux}

À partir du Magdalénien moyen, bien que les littoraux soient très éloignés de leur position actuelle, on assiste à une multiplication et une diversification d'indices d'exploitation du littoral par rapport aux périodes antérieures du Paléolithique récent (pour tout ce qui suit, nous renvoyons à Pétillon, 2016b et 2018, et aux références citées dans ces articles). Certes, comme précédemment au Paléolithique récent, les rivages atlantique et méditerranéen fournissent abondance de coquillages qui, une fois transformés en éléments de parure, circulent à l'intérieur des terres jusqu'à plusieurs centaines de kilomètres, tant depuis la Méditerranée que depuis l'Atlantique. Mais d'autres ressources, très rarement attestées dans les périodes antérieures, se rencontrent plus fréquemment, comme les vestiges de phoques, avec une petite dizaine de sites ayant livré des ossements isolés, dont une assez forte proportion de dents parfois transformées en parure, comme à Isturitz. Les cétacés sont attestés par quelques dents isolées, souvent percées et/ou sculptées (Duruthy, Mas d'Azil). Les os des grandes espèces ont également été utilisés pour la fabrication d'objets, essentiellement des pointes de projectile et des préhampes, dont on connaît environ 120 exemplaires sur le versant nord-pyrénéen (Pétillon, 2008 et 2013). La répartition des pièces suggère que ces ossements de cétacé sont d'origine atlantique et que les éléments ont ensuite diffusé vers le centre des Pyrénées suivant un réseau d'ouest en est, long de 300 km dans les Pyrénées (Pétillon, 2013: fig. 2). Les recherches sur ce thème sont en cours dans le cadre du projet ANR PaleoCet, coordonné par l'un d'entre nous (JMP).

\subsection{Les Pyrénées versant nord: une géogra- phie structurante}

En définitive, à l'ombre des Pyrénées, les dix derniers millénaires du Paléolithique récent-final apparaissent comme inégaux du point de vue de la qualité documentaire. À l'avenir, la recherche de sites post-DMG (transition Badegoulien - Magdalénien, Magdalénien inférieur) et de la transition Pléistocène - Holocène (Laborien) apparait comme une priorité parallèlement à la poursuite des opérations de terrain sur le Magdalénien moyen et supérieur et l'Azilien.

Malgré ces différents niveaux de résolution des données, il est possible de schématiquement subdiviser le versant nord en trois entités selon leurs trajectoires évolutives différentes au regard notamment du modèle aquitain (Fig. 8):

- un ensemble occidental fortement intégré à la plaine aquitaine au Pays basque;

- un ensemble central réunissant des influences atlantiques et aquitaines et des originalités régionales;

- un ensemble oriental ouvert à la fois aux influences occidentales et à celles de la Méditerranée, via l'arc catalo-languedocien. 


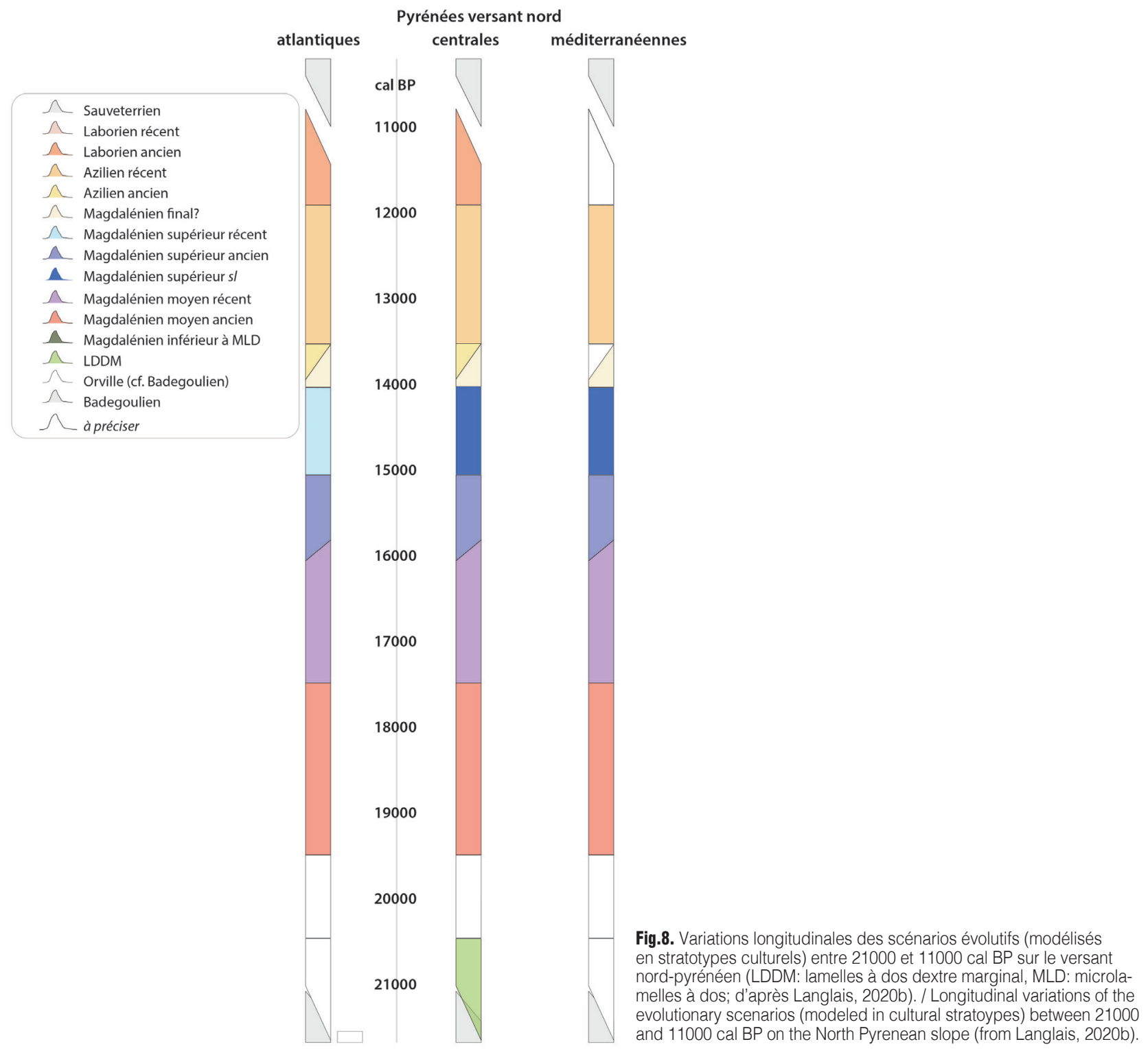

\section{CONCLUSION}

Ce bilan synthétique qui fait appel à de nombreux travaux publiés permet de souligner l'hétérogénéité des trajectoires culturelles du versant nord-pyrénéen entre 21000 et 11000 cal BP. Les variations longitudinales observées d'ouest en est soulèvent la question des facteurs ayant pu aboutir à ces phases d'unification et de régionalisation selon une progression non linéaire. Parallèlement, les circulations d'objets et de personnes entre les versants nord et sud ou entre le versant nord et l'Aquitaine, matérialisées notamment par la diffusion de matières premières lithiques et osseuses (e.g. Corchón et al., 2009; Sauvet et al., 2014; Pétillon et al. 2015; Sánchez de la Torre, 2015; Langlais et al., 2016; Lefebvre 2016; Sécher, 2017), permettent de souligner la forte inter-connectivité entre les communautés humaines au sein du Massif durant ces dix millénaires.

\section{REMERCIEMENTS}

Les auteurs remerciement C. Pailler, C. Renard, M. Jarry et $M$. Lejay pour l'organisation de la rencontre intitulée «Vivre pendant la dernière glaciation. Occupations humaines et paléoenvironnements: quelles spécificités dans les Pyrénées?» à Toulouse, en avril 2019. La plupart des résultats présentés ici ont été obtenus grâce au soutien financier de l'ANR, la Région Nouvelle-Aquitaine et la DRAC Nouvelle-Aquitaine.

\section{BIBLIOGRAPHIE}

Altuna, J., Merino, J.M. (dir.), 1984. El yacimiento prehistórico de la cueva de Ekain (Deba, Guipúzcoa), Eusko Ikaskuntza, San Sebastián.

Altuna, J., Baldeón, A., Mariezkurrena, K. (dir.), 1985. Cazadores magdalenienses en la cueva de Erralla (Cestona, Pais Vasco). Munibe Antropologia-Arkeologia, 37. 
Armendariz, A., 1994. Anton Koba: cazadores azilienses en la sierra de Aizkorri (Guipuzkoa). In: Balbin R., Bueno P., Congressos de Arqueologia Peninsular, Tomo 1, 297-310. Fondacion Rei Alfonso Henriques Ed.

Barandiaran, I., Cava, A., 1994. Zatoya, sitio magdaleniense de caza en medio pirenaico. In: Homenaje al Dr. Joaquín González Echegaray, 71-85. Ed. Museo y Centro de Investigación de Altamira.

Barbaza, M., 1996. Le Magdalénien supérieur final et l'Azilien dans les Pyrénées centrales. La grotte-abri du Moulin à Troubat (Hautes-Pyrénées) et son contexte. In: Delporte, H., Clottes, J. (dir.), Pyrénées préhistoriques arts et sociétés, 118e Congrès du CTHS, Pau 1993, 311-326. Ed. CTHS, Paris.

Barbaza, M., 2011. Environmental changes and cultural dynamics along the northern slope of the Pyrenees during the Younger Dryas. Quaternary International 242, 313-327.

Barbaza, M., Lacombe, S., 2005. L'Azilien pyrénéen: une culture originale? In: Jaubert. J., Barbaza, M. (dir.), Territoires, déplacements, mobilité, échanges durant la Préhistoire, 126e Congrès du CTHS, Toulouse, 2001, 421-428. Ed. CTHS, Paris.

Barshay-Szmidt, C., Costamagno, S., Henry-Gambier, D., Laroulandie, V., Pétillon, J.-M., Boudadi-Maligne, M., Kuntz, D., Langlais, M., Mallye, J.-B., 2016. New extensive focused AMS $14 \mathrm{C}$ dating of the Middle and Upper Magdalenian of the western Aquitaine/Pyrenean region of France (ca. 19-14 ka cal BP): Proposing a new model for its chronological phases and for the timing of occupation. Quaternary International 414, 62-91.

Bégouën, R., Fritz, C., Tosello, G., Clottes, J., Pastoors, A., Faist, F., 2009. Le sanctuaire secret des Bisons. II y a 14000 ans, dans la caverne du Tuc d'Audoubert. Association Louis Bégouën, Montesquieu-Avantès; Somogy Ed., Paris.

Bégouën, R., Pastoors, A., Clottes, J., 2019. La grotte d'Enlène, immersion dans un habitat magdalénien. Ed. In Fine, Paris

Buisson, D., Fritz, C., Kandel, D., Pinçon, G., Sauvet, G., 1996. Les contours découpés de têtes de chevaux

Briois, F., Vaquer, J., 2009. L'abri de Buholoup - De l'Épipaléolithique au Néolithique ancien dans le piedmont central des Pyrénées. In: Collectif (Eds.), De Méditerranée et d'ailleurs... Mélanges offerts à Jean Guilaine, 141-149. Archives d'Écologie Préhistoriques, Toulouse.

Calvet, M., Delmas, M., Gunnell, Y., Braucher, R., Bourlès, D., 2011. Recent advances in research on Quaternary glaciations in the Pyrenees, In: Ehlers, J., Gibbard, J., Hughes, P.D. (dir.), Quaternary Glaciations - Extent and Chronology. A Closer Look, 127-139. Elsevier (Developments in Quaternary Science, 15).

Cazals, N., 2000. Constantes et variations des traits techniques et économiques entre le Magdalénien inférieur et moyen: analyse des productions lithiques du Nord de la péninsule ibérique. Thèse de Doctorat, Université de Paris I Panthéon-Sorbonne.

Cazals, N., Langlais, M., 2005-2006. La place d'Ekain (couche VII) au sein du Magdalénien basco-cantabrique: nouvelles contributions sur l'organisation des productions lithiques. In: Homenaje a Jesús Altuna t. I, Munibe Antropologia-Arkeologia 57,117-191.

Cazals, N., Urquijo, J.E., Terradas, X. (dir.), 2007. Frontières naturelles et frontières culturelles dans les Pyrénées préhistoriques, Table ronde de Tarascon/Ariège, 2004. Ediciones Universidad de Cantabria, Santander.

Corchon, M.S., Tarriño, A., Martinez, X., 2009. Mobilité, territoires et relations culturelles au début du Magdalénien moyen cantabrique: nouvelles perspectives. In: Djindjian, F., Kozlows- ki, J., Bicho N. (dir.), Le concept de territoire dans le Paléolithique supérieur européen, Actes du 15e congrès de I'UISPP (Lisbonne, 2006), 217-230. Archaeopress, Oxford (BAR, International Series 1938).

Costamagno, S., Laroulandie, V., 2004. L'exploitation des petits vertébrés dans les Pyrénées françaises du Paléolithique au Mésolithique: un inventaire taphonomique et archéozoologique. In: Brugal, J.-P., Desse, J. (dir.), Petits animaux et sociétés humaines. Du complément alimentaire aux ressources utilitaires, XXIVe rencontres internationales d'archéologie et d'histoire d'Antibes, 403-416. Ed. APDCA.

Costamagno, S., Barshay-Szmidt, C., Kuntz, D., Laroulandie, V., Pétillon, J.-M., Boudadi-Maligne, M., Langlais, M., Mallye, J.-B., Chevallier, A., 2016. Reexamining the timing of reindeer disappearance in southwestern France in the larger context of the late glacial faunal turnover. Quaternary International, 414, 34-61.

Couraud C., 1985. L'art azilien: origine, survivance, Gallia Préhistoire, supplément XX. CNRS, Paris.

Dachary, M., 2002. Le Magdalénien des Pyrénées occidentales. Thèse de Doctorat, Université de Paris X, Nanterre, spécialité Préhistoire. Ex. multigraph.

Delmas, M., 2015. The Last Maximum ice extent and subsequent deglaciation of the Pyrenees: an overview of recent research. Cuadernos de Investigación Geográfica 41(2), 359-387.

Ducasse, S., Castel, J.-C., Chauvière, F.-X., Langlais, M., Camus, H., Morala, A., Turq, A., 2011. Le Quercy au cœur du Dernier Maximum glaciaire. La couche 4 du Petit Cloup Barrat et la question de la transition badegoulo-magdalénienne. Paleo 22, 101-154.

Ducasse, S., Langlais, M., 2019. Twenty years on, a new date with Lascaux reassessing the chronology of the cave's paleolithic occupations through new 14c AMS dating. Paleo 30(1), 130-147.

Fat Cheung, C., 2015. L'Azilien pyrénéen parmi les sociétés du Tardiglaciaire ouest-européen: apport de l'étude des industries lithiques. Ph.D., Université de Toulouse.

Fat Cheung, C., 2020. Lithic Perspectives on the Late Upper Palaeolithic in the French Pyrenees, Quaternary International 564, 16-36.

Fat Cheung, C., Langlais, M., sous presse. El Paleolítico final del suroeste de Francia, desde los Pirineos hasta el Atlántico. In: Roman-Monroig D. (dir.), Las facies microlaminares del final del Paleolitico en el mediterraneo ibérico y el valle del Ebro, Workshop'2018, Vilafranca (Castellon), 5-7 de octubre.

Jalut, G., Andrieu, V., Delibrias, G., Fontugne, M., Pagès, P., 1988. Palaeoenvironment of the valley of Ossau (Western French Pyrenees) during the last 27,000 years. Pollens et spores 30, 357-394.

Jalut, G., Montserrat, J., Fontugne, M., Delibrias, G., Vilaplana, J.M., Julia, R., 1992. Glacial to interglacial vegetation changes in the northern and southern Pyrenees: deglaciation, vegetation cover and chronology. Quaternary Science Reviews 11, 449-480.

Lacombe, S., 1998. Préhistoire des groupes culturels au Tardiglaciaire dans les Pyrénées centrales. Apports de la technologie lithique. Thèse de Doctorat, Université de Toulouse-Le Mirail.

Langlais, M., 2007. Dynamiques culturelles des sociétés magdaléniennes dans leurs cadres environnementaux. Enquête sur 7000 ans d'évolution de leurs industries lithiques entre Rhône et Ėbre. Thèse de doctorat, Université de Toulouse-Le Mirail et Universitat de Barcelona. 
Langlais, M., 2010. Les sociétés magdaléniennes de l'isthme pyrénéen. CTHS, Paris (Documents préhistoriques, 26).

Langlais, M., 2018. Le Magdalénien supérieur: une technologie de pointes. In: Averbouh A., Bonnet-Jacquement P., Cleyet-Merle J.-J. (Eds.), L'Aquitaine à la fin des temps glaciaires - Aquitaine at the end of the Ice Age. Les sociétés de la transition du Paléolithique final au début du Mésolithique dans l'espace Nord aquitain. Paleo nº spécial, 97-108.

Langlais, M., 2020a. Des segments chronoculturels au modèle archéo-stratigraphique du Magdalénien dans le sud-ouest français $(21000-16000$ cal BP). In: Straus, L.G., Langlais M. (dir.), Magdalenian Chrono-stratigraphic Correlations and Cultural Connections between Cantabrian Spain and Southwest France....and Beyond, actes du colloque XVIIIle congrès de I'UISPP et séance de la Société préhistorique Française, Paris, juin 2016, 109-136. Séances de la Société préhistorique française.

Langlais, M., 2020b. Une anthologie du Paléolithique récent-final en France méridionale (21-11 Ky cal BP). Technologie lithique culturelle et dynamiques spatio-temporelles, mémoire d'Habilitation à diriger des recherches. Université de Bordeaux, 2 volumes, inédit.

Langlais, M., Pétillon, J.-M., De Beaune, S. A., Cattelain, P., Chauvière, F.-X., Letourneux, C., Szmidt, C., Bellier, C., Beukens, R., David, F., 2010. Une occupation de la fin du dernier maximum glaciaire dans les Pyrénées: le Magdalénien inférieur de la grotte des Scilles (Lespugue, Haute-Garonne). Bulletin de la Société Préhistorique Française 107(1), 5-51.

Langlais, M., Sécher, A., Caux, S., Delvigne, V., Gourc, L., Normand, C., Sanchez De La Torre, M., 2016. Lithic Tool Kits: A Metronome of the Evolution of the Magdalenian in Southwest France (19,000-14,000 cal BP). Quaternary International 414, 92-107.

Langlais, M., Pétillon, J.-M., Sécher, A., 2017. Les débuts du Magdalénien dans le sud-ouest français: témoignages croisés des équipements lithiques et osseux. In: Bourdier C., Chehmana L., Malgarini R., Poltowicz-Bobak M. (dir.), L'essor du Magdalénien: aspects culturels, symboliques et techniques des faciès à navettes et à Lussac-Angles, Actes de la séance de la SPF de Besançon, 17-19 octobre 2013, Paris, 209-234. Séance de la Société préhistorique française.

Langlais, M., Pétillon, J.M., 2019. Les Pyrénées, une frontière pré-historiographique pour le Magdalénien? Réflexions à partir du Magdalénien moyen de la Grotte Tastet (Sainte-Colome, Pyrénées-Atlantiques). In: Deschamps, M., Costamagno, S., Milcent, P.-Y., Pétillon, J.-M., Renard, C., Valdeyron, N. (dir.), La conquête de la montagne, des premières occupations humaines à l'anthropisation du milieu, actes du 142e congrès du CTHS (Pau, 2017). CTHS, Paris. Disponible depuis: https:// books.openedition.org/cths/6182?lang=es

Langlais, M., Naudinot, N., Pasty, J.F., Marquebielle, B., Fat Cheung, C., Bonnet-Jacquement, P., Detrain, L., 2019. D'un Massif à l'autre: synthèse sur le Laborien entre France méridionale et atlantique. In: Fagnart, J.-P., Mevel, L., Valentin, V., Weber, M.-J. (dir.), L'Europe du nord-ouest autour de 9600 Cal. B.C.: quels changements?, Actes du XXVIII Congrès Préhistorique de France d'Amiens, 30 mai au 4 juin 2016, 349-362. Mémoire de la Société Préhistorique Française.

Laroulandie, V., 2007. Les restes aviaires des niveaux aziliens de la grotte-abri du Moulin (Troubat, Hautes-Pyrénées): Paléoenvironnement et modalités d'exploitation. Bulletin de Préhistoire du Sud-Ouest 14(1), 19-29.

Laroulandie, V., 2016. Hunting fast-moving, low-turnover small game: The status of the snowy owl (Bubo scandiacus) in the Magdalenian. Quaternary International 414, 174-197.

Laroulandie, V., Elorza, M., Berganza, E., 2016. Les oiseaux marins du Magdalénien supérieur de Santa Catalina (Lekeitio, Bis- caye, Espagne): approches taphonomique et archéozoologique. In: Dupont C., Marchand G. (dir.), Archéologie des chasseurs-cueilleurs maritimes. De la fonction des habitats à l'organisation de l'espace littoral, Actes de la séance de la Société préhistorique française de Renne, Seapeople 2014, 10-11 avril 2014, 37-57. Société préhistorique française, Séances SPF, Paris.

Laroulandie, V., Costamagno, S., Langlais, M., Pétillon, J.-M., 2017. L'œuf ou la poule? Retour sur le projet Magdatis, Le Magdalénien de la façade atlantique face aux changements environnementaux, Quaternaire 28(2), 277-283.

Lefebvre, A., 2016. Les stratégies d'adaptation des sociétés pyrénéennes entre 19 et 14 ka cal BP. Étude biométrique et techno-économique comparée sur l'exploitation du bois de cerf et du bois de renne autour des Pyrénées au Magdalénien moyen et supérieur. Thèse de doctorat, Université de Bordeaux.

Lefebvre A., Marín-Arroyo A.B., Alvarez-Fernandez E., De la Rasilla Vives M., Duarte Matías E., Cueto E., Tapia J., Berganza E., Pétillon J.M. (sous presse) - Interconnected Magdalenian societies as revealed by the circulation of whale bone artefacts in the Pyreneo-Cantabrian region, Quaternary Science Reviews 251, doi.org/10.1016/j.quascirev.2020.106692.

Mangado, J. (dir.), 2018. Montlleó: el paleolític superior a Cerdanya. Resultats de 20 anys de recerca arqueològica. Homenatge a Oriol Mercadal Fernàndez, Universitat de Barcelona, Societat Catalana d'Arqueologia (Sèrie Vària del SERP, 1).

Marquebielle, B., 2018. Une rupture dans le travail des matières osseuses entre le Paléolithique final et le Mésolithique? Quelques éléments de réflexion à partir de sites Nord aquitains. In: Averbouh, A., Bonnet-Jacquement, P., Cleyet-Merle, J.J., (eds), L'Aquitaine à la fin des temps glaciaires. Les sociétés de la transition du Paléolithique final au début du Mésolithique dans l'espace Nord aquitain, actes du colloque en hommage à Guy Célérier, Les Eyzies, juin 2015. Paleo n spécial, 137-146.

Martzluff, M., 2005. Evolucio de la talla litica a la sequencia aziliana de la Balma de la Margineda. In: Guilaine, J., Barbaza, M., Martzluff, M. (dir.), Les excavacions a la Balma de la Margineda 1979-1991, 574-598. Principat d'Andorra.

Martzluff, M., 2009. L'Azilien pyrénéen entre Garonne et Ebre: un état de la question. In: Fullola, J.M., Valdeyron, N., Langlais, M. (eds.), Els Pirineus i les arees circumdants durant el Tardiglacial. Mutacions i filiacions tecnoculturals, evolucio paleoambiental, Homenatge al Professor Georges Laplace, XIVe colloqui internacional d'arqueologia de Puigcerdà, 10-12 novembre, 2006, 375-422. Institut d'Estudis Ceretans.

Mevel, L., 2017. Des sociétés en mouvement. Évolution des sociétés magdaléniennes et aziliennes des Alpes du Nord françaises. CTHS Éd., Paris (Documents Préhistoriques 34).

Naudinot, N., Fagnart, J.P., Langlais, M., Mevel, L., Valentin, B., 2019. Les dernières sociétés du Tardiglaciaire et des tout débuts de l'Holocène en France. Bilan d'une trentaine d'années de recherche, Gallia Préhistoire 58, 5-45.

Pétillon, J.-M., 2006. Des Magdaléniens en armes. Technologie des armatures de projectile en bois de Cervidé du Magdalénien supérieur de la grotte d'Isturitz (Pyrénées-Atlantiques). CEDARC, Treignes (Artefacts 10).

Pétillon, J.-M., 2008. First evidence of a whale-bone industry in the western European Upper Paleolithic: Magdalenian artifacts from Isturitz (Pyrénées-Atlantiques, France). Journal of Human Evolution 54(5), 720-726.

Pétillon, J.-M., 2013. Circulation of whale-bone artifacts in the northern Pyrenees during the Late Upper Paleolithic. Journal of Human Evolution 65(5), 525-543. 
Pétillon, J.-M., 2016a. Technological Evolution of Hunting Implements among Pleistocene Hunter-Gatherers: Osseous Projectile Points in the Middle and Upper Magdalenian (19-14 ky cal BP). Quaternary International 414, 108-134

Pétillon, J.-M., 2016b. Vivre au bord du golfe de Gascogne au Paléolithique supérieur récent: vers un nouveau paradigme / Life on the shore of the Bay of Biscay in the Late Upper Paleolithic: towards a new paradigm. In: Dupont, C., Marchand, G. (dir.), Archéologie des chasseurs-cueilleurs maritimes. De la fonction des habitats à l'organisation de l'espace littoral / Archaeology of maritime hunter-gatherers. From settlement function to the organization of the coastal zone, 23-36. Société Préhistorique Française, Paris (Séances de la Société préhistorique française, 6).

Pétillon, J.-M., 2018. Échos de l'océan: phoques et baleines en Europe au Paléolithique récent. In: Cattelain, P., Gillard, M., Smolderen, A. (dir.), Disparus? Les mammifères au temps de Cro-Magnon en Europe, 335-354. Cedarc (catalogues d'exposition), Treignes.

Pétillon, J.-M., Averbouh, A., 2013. L'industrie osseuse du Solutréen au Magdalénien moyen dans le Bassin parisien. In: Bodu, P., Chehmana, L., Klaric, L., Mevel, L., Soriano,S., Teyssandier, N. (dir.), Le Paléolithique supérieur ancien de l'Europe du Nord-Ouest, actes du colloque (Sens, 15-18 avril 2009), 143-158. Société préhistorique française, Paris (Mémoire, 56).

Pétillon, J.-M., Ducasse, S., 2012. From flakes to grooves: a technical shift in antlerworking during the Last Glacial Maximum in southwest France. Journal of Human Evolution 62(4), 435-465.

Pétillon, J.-M., Langlais, M., De Beaune, S.A., Chauvière, F.-X., Letourneux, C., Szmidt, C., Beukens, R., David, F., 2008. Le Magdalénien de la grotte des Scilles (Lespugue, Haute-Garonne). Premiers résultats de l'étude pluridisciplinaire de la collection Saint-Périer. Antiquités nationales 39, 57-71.

Pétillon, J.-M., Langlais, M., Kuntz, D., Normand, C., Barshay-Szmidt, C., Costamagno, S., Delmas, M., Laroulandie, V., Marsan, G., 2015. The human occupation of the northwestern Pyrenees in the Late Glacial: new data from the Arudy basin, lower Ossau valley. Quaternary International 364, 126-143.

Pétillon, J.-M., Laroulandie, V., Costamagno, S., Langlais, M., 2016. Testing environmental determinants in the cultural evolution of hunter-gatherers: a three-year multidisciplinary project on the occupation of the western Aquitaine basin during the Middle and Upper Magdalenian (19-14 kyr cal BP). Quaternary International 414, 1-8.

Pétillon, J.-M., Laroulandie, V., Costamagno, S., Langlais, M. eds., 2016. Magdatis project: Hunter-gatherers and environmental change in the Aquitaine basin during the Magdalenian. Special section of Quaternary International 414, 1-197.

Pétillon, J.-M., Laroulandie, V., Boudadi-Maligne, M., Dumontier, P., Ferrier, C., Kuntz, D., Langlais, M., Mallye, J.-B., Mistrot, V., Normand, C., Rivero Vilá, O., Sánchez De La Torre, M., 2017. Occupations magdaléniennes entre 20000 et 15000 cal BP dans le piémont pyrénéen: la séquence paléolithique du sondage 4 de la grotte de Laa 2 (Arudy, Pyrénées-Atlantiques). Gallia Préhistoire 57, 65-126.

Rasmussen, S.O., Bigler, M., Blockley, S.P., Blunier, T., Buchardt, S.L., Clausen, H.B., Cvijanovic, I., Dahl-Jensen, D., Johnsen, S.J., Fischer, H., Gkinis, V., Guillevic, M., Hoek, W.Z., Lowe, J.J., Pedro, J.B., Popp, T., Seierstad, I.K., Steffensen, J.P., Svensson, A.M., Vallelonga, P., Vinther, B.M., Walker, M.J., Wheatley, J.J., Winstrup, M., 2014. A stratigraphic framework for abrupt climatic changes during the Last Glacial period based on three synchronized Greenland ice-core records: refining and extending the INTIMATE event stratigraphy. Quaternary Science Reviews 106, 14-28.
Roman, D., Nadal, J., Domingo, I., Garcia-Argüelles, P., Lloveras, L., Fullola, J.M., 2016. La fin du Paléolithique dans la Catalogne méridionale ibérique revisitée: nouvelles réponses pour anciennes questions. L'Anthropologie 120, 610-628.

Sacchi, D., 1986. Le Paléolithique supérieur du Languedoc occidental et du Roussillon. Gallia Préhistoire XXle suppl. CNRS, Paris.

Sánchez De La Torre M., 2015. Las sociedades cazadoras-recolectoras del Paleolítico Superior final pirenaico: territorios económicos y sociales. Thèse de doctorat, Universitat de Barcelona.

Sánchez De La Torre, M., Mangado, X., Le Bourdonnec, F.X., Gratuze, B., Langlais, M., Mercadal, O. (†), Fullola, J.M., 2018. Tracing prehistoric past human routes in the Pyrenees: new data about chert procurement at Montlleó open-air site (Prats i Sansor, Spain). $18^{\text {th }}$ UISPP World Congress, Paris.

Sauvet, G., Fritz, C., Fortea, Perez, J., Tosello, G., 2014. Fluctuations des échanges symboliques au Paléolithique supérieur en France et dans le Nord de l'Espagne. In: Jaubert J., Fourment, N., Depaepe, P. (dir.), Transitions, ruptures et continuités en Préhistoire, Actes du 27e Congrès préhistorique de France, Bordeaux-Les Eyzies, juin 2010, 403-415. Société Préhistorique Française, Paris.

Sécher, A., 2017. Traditions techniques et paléogéographie du Magdalénien moyen ancien dans le Sud-Ouest de la France (19000-17500 cal. BP). Des groupes humains à plusieurs visages? Thèse de doctorat, Université de Bordeaux.

Sécher, A., 2020. Le Magdalénien moyen ancien dans le sudouest de la France. Essai d'approche paléogéographique des traditions culturelles. In: Straus, L.G., Langlais, M. (dir.), Magdalenian chrono-stratigraphic correlations and cultural connections between Cantabrian Spain and Southwest France... and beyond. Actes du colloque XVIIIe congrès de I'UISPP et séance de la Société préhistorique Française, Paris, juin 2016, 41-64. Séance de la Société préhistorique française.

Simonnet, R., 1967. L'abri sous roche Rhodes II et la question de l'Azilien dans les Pyrénées françaises. Bulletin de la Société préhistorique française, Études et travaux 64(1), 175-186.

Simonnet, R., 1982. Grandes lames de silex dans le Paléolithique supérieur des Pyrénées centrales. Essai sur des documents marginaux. Bulletin de la Société préhistorique de l'Ariège XXXVII, 61-105

Soto, A., Alday, A., Montes, L., Utrilla, P., Perales, U., Domingo, R., 2015. Epipalaeolithic assemblages in the Western Ebro Basin (Spain): The difficult identification of cultural entities, Quaternary International 364, 144-152.

Straus, L.G., Langlais, M. (dir.), 2020. Magdalenian chrono-stratigraphic correlations and cultural connections between Cantabrian Spain and Southwest France....and beyond. Actes du colloque XVIIle congrès de I'UISPP et séance de la Société préhistorique Française, Paris, juin 2016. Séance de la Société Préhistorique Française 15

Tomasso, A., Fat Cheung, C., Fornage-Bontemps, S., Langlais, M., Naudinot, N., 2018. Winter is coming: What happened in western European mountains between 12.9 and $12.6 \mathrm{ka} \mathrm{cal}$. BP (beginning of the GS1), Quaternary International 465, 210-221.

Utrilla, P., Mazo, C., 1996. Le versant sud des Pyrénées. In: Delporte, H., Clottes, J. (dir.), Pyrénées préhistoriques arts et sociétés, 118e Congrès du CTHS, Pau, 1993, 60-69. Ed. CTHS, Paris.

Utrilla, P., Laborda, R., 2018. La cueva de Chaves (Bastaras, Huesca): 15000 años de ocupación prehistórica. Trabajos de Prehistoria 75(2), 248-269. 
